\title{
Grasp-to-retract modification of the tulip-bundle technique in forward and retroflexed position for difficult hemostatic therapy in the sigmoid colon
}

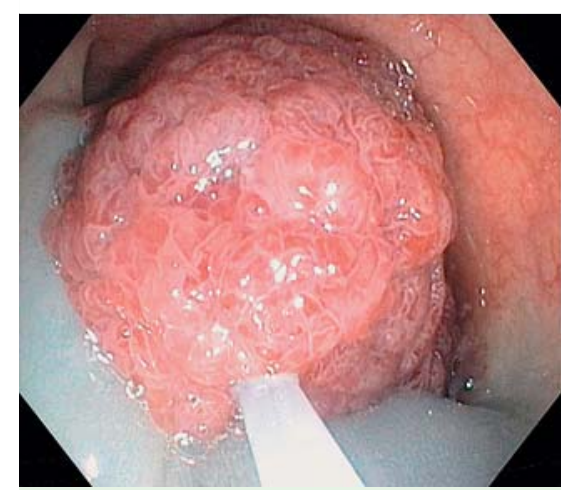

Fig. 1 Endoscopic mucosal resection of a 7-cm, type 0 -Is lesion in the distal sigmoid colon.

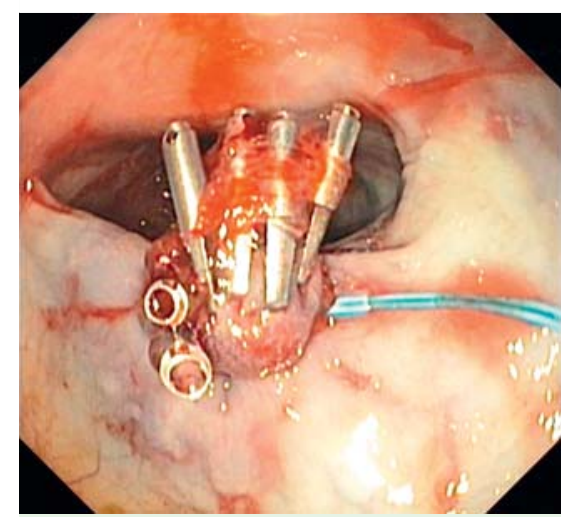

Fig. 4 Using a double-channel colonoscope, a grasping forceps was used to retract the defect while a detachable snare was positioned underneath the clips and closed, resulting in immediate hemostasis.

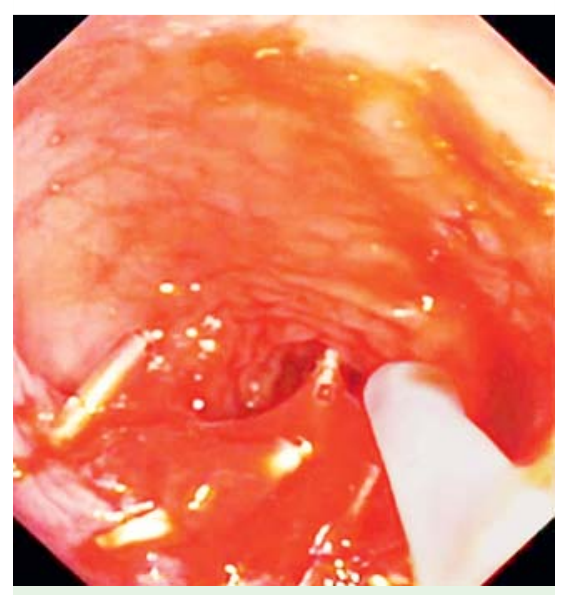

Fig. 2 After complete resection, a $4 \times 4 \mathrm{~cm}$ mucosal defect over a colonic fold was apparent, with diffuse oozing but no visible vessels. The defect was closed using hemostatic clips, but diffuse oozing persisted between the clips.

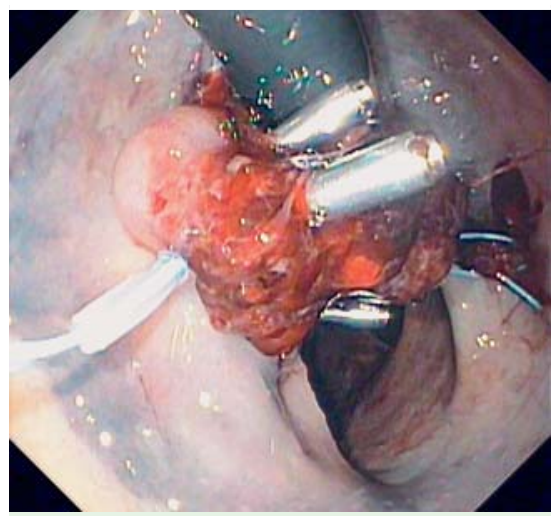

Fig. 5 In a second colonoscopy for hematochezia 4 hours later, oozing was observed from the proximal border of the defect. The same grasp-to-retract and tulip-bundle technique previously described was performed in retroflexed position in the sigmoid colon, resulting in prompt hemostasis.

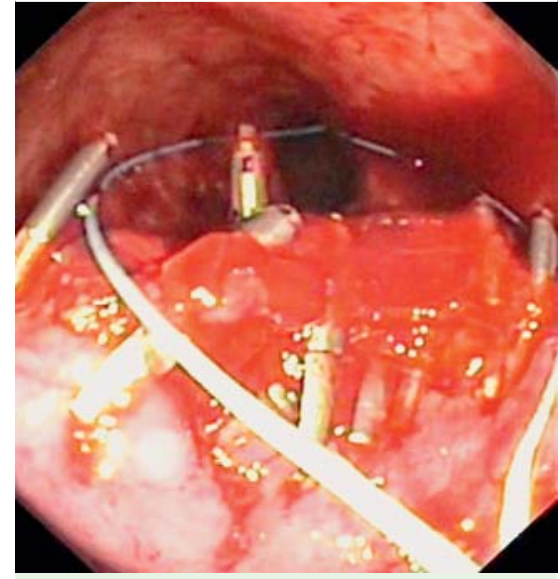

Fig.3 Attempts to place a detachable snare underneath the clips, in order to perform the tulip-bundle technique, were unsuccessful because of the large diameter of the defect with clips and its position over the colonic fold.

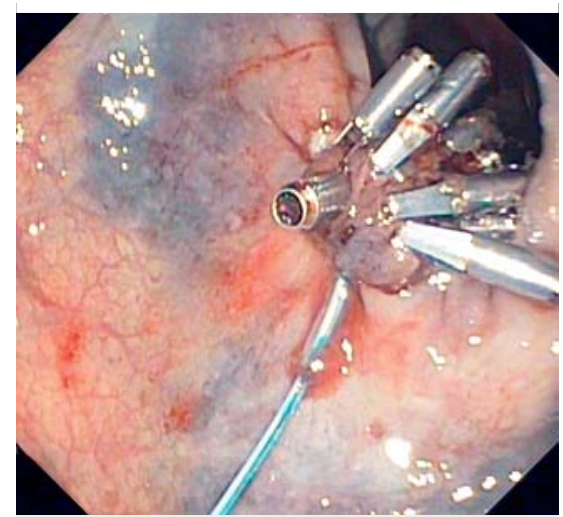

Fig. 6 Final defect, in forward-viewing position, after the two tulip-bundle procedures, showing definitive hemostasis.
A 60-year-old woman with no relevant medical history underwent endoscopic mucosal resection (EMR) of a 7-cm 0-Is lesion in the distal sigmoid colon. A solution of saline, indigo carmine, and 1/100 000 adrenaline was injected into the submucosa, and piecemeal snare resection was performed ( $\bullet$ Fig. 1). Persistent oozing occurred during EMR and was partially controlled by subsequent submucosal injections and resections. After complete resection, a $4 \times 4 \mathrm{~cm}$ mucosal defect over a colonic fold could be seen, with diffuse oozing but no visible vessels.

The defect was closed using hemostatic clips, but diffuse oozing persisted between the clips ( $\bullet$ Fig. 2). Attempts to place a detachable snare (MAJ-254; Olympus, Tokyo, Japan) underneath the clips, in order to perform the tulip-bundle technique, were unsuccessful because of the large diameter of the defect with clips and its position over the fold ( $\bullet$ Fig.3, - Video 1). Therefore, a double-channel colonoscope (GIF 2T160I; Olympus) was used, and a grasping forceps was used to retract the defect while the detachable snare was positioned underneath the clips, resulting in immediate hemostasis (॰ Fig. 4, $\diamond$ Video 2).

Despite initial hemostasis, the patient presented with hematochezia 4 hours later. Recurrent oozing from the proximal border of the mucosal defect, which had not been entrapped by the detachable snare, was observed and could not be 


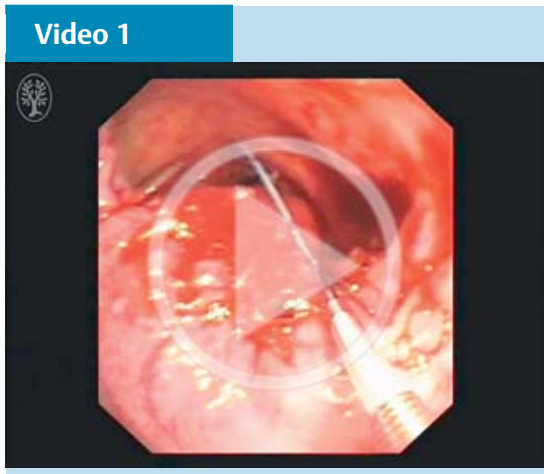

Attempts to perform the tulip-bundle technique to treat diffuse oozing from a large endoscopic mucosal resection defect closed with clips were unsuccessful because of the large diameter of the defect with clips and its position over a colonic fold.

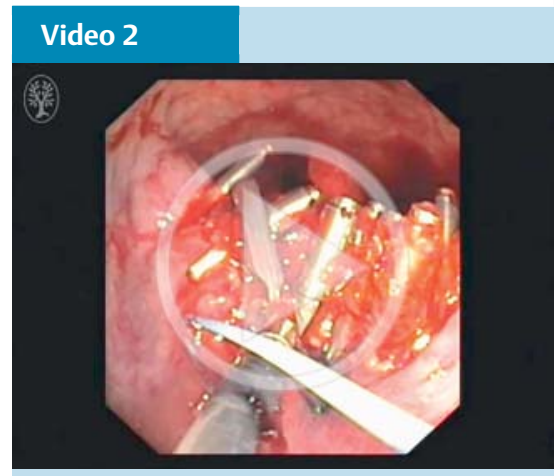

A double-channel colonoscope was used to retract the defect with a grasping forceps while a detachable snare was positioned underneath the clips and closed, resulting in instant hemostasis.

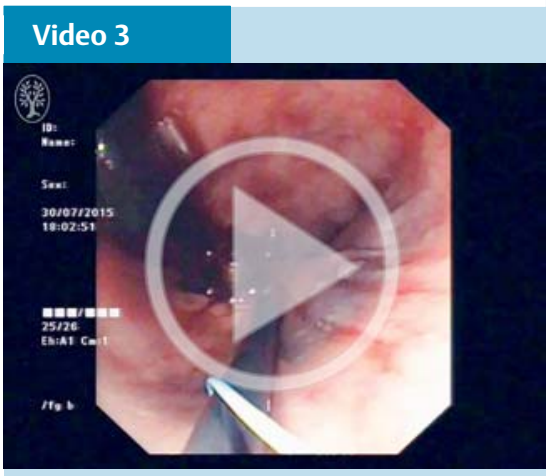

The same grasp-to-retract and tulip-bundle technique was performed in the retroflexed position in the sigmoid colon to treat bleeding that occurred from the proximal border of the defect 4 hours later. treated with further clipping. With the endoscope in the retroflexed position, the tulip-bundle technique was attempted but was, again, unsuccessful. Using the double-channel colonoscope in the retroflexed position, and the same grasp-to-retract and tulip-bundle technique, definitive hemostasis was achieved ( $\bullet$ Fig. 5 , - Fig. 6, V Video 3).

Histology revealed a tubulovillous adenoma with high grade dysplasia.

Detachable snares have various indications that include assisting polypectomy, resecting submucosal tumors [1], and performing full-thickness resections [2]. The tulip-bundle technique involves the snare entrapping the clips to achieve hemostasis [3] or to close perforations [4]. This grasp-to-retract modification, which has been described previously for other techniques [5], can assist the tulipbundle technique in difficult procedures.

Endoscopy_UCTN_Code_CPL_1AJ_2AD

Competing interests: None
Rolando Pinho', Joana Silva',

Ana Ponte ${ }^{1}$, Jaime Rodrigues ${ }^{1}$,

Iolanda Ribeiro', Maria Conceição Lucas $^{2}$, João Carvalho ${ }^{1}$

${ }^{1}$ Gastroenterology Department, Centro Hospitalar de Vila Nova de Gaia, Vila Nova de Gaia, Portugal

2 Surgery Department, Centro Hospitalar de Vila Nova de Gaia, Vila Nova de Gaia, Portugal

\section{References}

1 Veloso R, Pinho R, Rodrigues A et al. Endoloop ligation ("loop-and-let-go") of a large ileal lipoma by balloon-assisted enteroscopy. Endoscopy 2012; 44 (Suppl. 02): E176

2 Pinho R, Oliveira M, Mascarenhas-Saraiva M. Endoscopic full-thickness resection of an inverted colonic diverticulum with intraepithelial neoplasia using the ligate-and-let-go technique. Clin Gastroenterol Hepatol. In press 2015. DOI: 10.1016/j.cgh.2015.08.007

3 Lee JH, Kim BK, Seol DC et al. Rescue endoscopic bleeding control for nonvariceal upper gastrointestinal hemorrhage using clipping and detachable snaring. Endoscopy 2013; 45: 489-492
4 Mocciaro F, Curcio G, Tarantino I et al. Tulip bundle technique and fibrin glue injection: unusual treatment of colonic perforation. World J Gastroenterol 2011; 17: 1088 - 1090

5 Ponte A, Pinho R, Vale $S$ et al. Resection of a large ileal lipoma exhibiting ball-valve prolapse into the cecum with a "grasp-to-retract, ligate, unroof, and let-go" technique. Endoscopy 2015; 47 (Suppl. 01): E215-216

Bibliography

DOI http://dx.doi.org/

10.1055/s-0034-1393393

Endoscopy 2015; 47: E554-E555

(c) Georg Thieme Verlag KG

Stuttgart · New York

ISSN 0013-726X

\section{Corresponding author}

\section{Rolando Taveira Pinho, MD}

Serviço de Gastrenterologia

Centro Hospitalar de Vila Nova de Gaia

Rua Conceição Fernandes

Vila Nova de Gaia 4434-502

Portugal

Fax: +351-227-868369

rolandopinho@gmail.com 